\title{
ON EIGENVALUE DISTRIBUTIONS FOR ELLIPTIC OPERATORS WITHOUT SMOOTH COEFFICIENTS
}

\author{
BY RICHARD BEALS ${ }^{1}$
}

Communicated by F. Browder, March 10, 1966

1. Introduction. An asymptotic formula for the eigenvalues of a self-adjoint realization of an elliptic operator on a bounded region has been obtained in various cases by a number of authors; see [1], [4] and the references there. In each case the method of proof demands more regularity of the coefficients of the operator (at least when the order of the operator is low relative to the dimension of the space) than should be necessary for validity of the formula. The purpose of this note is to derive the formula under minimum assumptions on the coefficients, in two cases: the Dirichlet realization in a compact manifold with boundary, and the unique realization in a compact manifold without boundary. We use the known results for operators with smooth coefficients and a simple abstract approximation method based on the "minimax" formula for eigenvalues.

2. Abstract eigenvalue estimates. Let $H$ be a separable complex Hilbert space with inner product $(u, v)$ and norm $\|u\|$. If $S$ is a compact operator in $H$, we denote by $\left\{\mu_{j}(S)\right\}$ the sequence of eigenvalues of $S S^{*}$, with $\mu_{1}(S) \geqq \mu_{2}(S) \geqq \cdots$.

Suppose that $S$ is a $1-1$ compact normal operator in $H$. The range $R(S)$ can be considered as a Hilbert space $K$ with inner product $\langle u, v\rangle=\left(S^{-1} u, S^{-1} v\right)$ and norm $|u|$. Let $T$ be a second operator in $H$ with $R(T) \subseteq K$. If $J$ denotes the natural injection of $K$ into $H$, then $S=J S_{1}$ and $T=J T_{1}$ with $S_{1}$ and $T_{1}$ operators from $H$ to $K$. Since $T_{1}$ is closed, hence continuous, and $J$ is compact, it follows that $T$ is compact. We can estimate the eigenvalues $\mu_{j}(T)$ as follows:

Lemma 1. For all $j=1,2, \cdots$,

$$
\mu_{j}(T) \leqq\left(\left\|T_{1}\right\|+\left\|S_{1}\right\|\right)\left\|T_{1}-S_{1}\right\| \mu_{j}(S)+\mu_{j}(S) .
$$

Proof. Let $\left\{\phi_{j}\right\}$ be a complete orthonormal sequence in $H$ consisting of eigenvectors of $S$, with corresponding eigenvalues $\left\{\lambda_{j}\right\}$ satisfying $\left|\lambda_{1}\right| \geqq\left|\lambda_{2}\right| \geqq \ldots$. Let $H_{j}$ be the closed subspace of $H$ spanned by $\left\{\phi_{k} ; k \geqq j\right\}$. Applying the "minimax" formula [5, Theorem X.4.3] to the positive compact operator $T T^{*}$ we have

1 Research partially supported by the Air Force Office of Scientific Research Grant AF-AFOSR-736-65. 


$$
\begin{aligned}
\mu_{j}(T) & \leqq \max _{u \in H_{j},\|u\|=1}\left(T T^{*} u, u\right) \\
& =\max _{u \in H_{j},\|u\|=1}\left\{\left(S S^{*} u, u\right)+\left(\left(T T^{*}-S S^{*}\right) u, u\right)\right\} \\
& \leqq \mu_{j}(S)+\max _{u \in H_{j},\|u\|=1}\left|\left(\left(T T^{*}-S S^{*}\right) u, u\right)\right| .
\end{aligned}
$$

Now $S^{*}=S_{1}^{*} J^{*}$ and $T^{*}=T_{1}^{*} J^{*}$, so

$$
\begin{aligned}
\left|\left(\left(T T^{*}-S S^{*}\right) u, u\right)\right| & \leqq\left|\left(\left(T^{*}-S^{*}\right) u, T^{*} u\right)\right|+\left|\left(S^{*} u,\left(T^{*}-S^{*}\right) u\right)\right| \\
& \leqq\left(\left\|T_{1}^{*}\right\|+\left\|S_{1}^{*}\right\|\right)\left\|T_{1}^{*}-S_{1}^{*}\right\|\left|J^{*} u\right|^{2} \\
& =\left(\left\|T_{1}\right\|+\left\|S_{1}\right\|\right)\left\|T_{1}-S_{1}\right\|\left|J^{*} u\right|^{2} .
\end{aligned}
$$

The vectors $\left\{\lambda_{k} \phi_{k}\right\}$ are a complete orthonormal sequence in $K$. It is easily seen that $J^{*} \phi_{j}=\lambda_{j}^{2} \phi_{j}$, so $\left|J^{*} \phi_{j}\right|=\left|\lambda_{j}\right|$. Since $\left|\lambda_{1}\right| \geqq\left|\lambda_{2}\right| \geqq \cdots$, it follows that for $u \in \mathcal{F}_{j},\left|J^{*} u\right| \leqq\left|\lambda_{j}\right|\|u\|$. But $\left|\lambda_{j}\right|^{2}=\mu_{j}(S)$, so the desired result follows from (1) and (2).

In particular, let $A=S_{1}^{-1}: K \rightarrow H$ and suppose $B: K \rightarrow H$ has norm $\|B\|=\epsilon<1$. Then $\left\|B A^{-1}\right\|=\epsilon$ in $K$, so $A+B=\left(I+B A^{-1}\right) A$ has an inverse $T_{1}: H \rightarrow K$. Furthermore $\left\|T_{1}\right\|=\left\|\left(I+B A^{-1}\right)^{-1}\right\| \leqq(1-\epsilon)^{-1}$, and $T_{1}-S_{1}=S_{1}\left(\left(I+B A^{-1}\right)^{-1}-I\right)$ has norm $\left\|T_{1}-S_{1}\right\| \leqq \epsilon(1-\epsilon)^{-1}$. Set $T=J T_{1}$. If $T$ is normal then by Lemma 1 ,

$$
\begin{aligned}
\mu_{j}(T) & \leqq\left[1+\epsilon(1-\epsilon)^{-1}\left(1+(1-\epsilon)^{-1}\right)\right] \mu_{j}(S) \\
& =(1-\epsilon)^{-2} \mu_{j}(S) .
\end{aligned}
$$

Conversely, let $K_{1}$ be the space $K$ with the inner product induced by $T$. Then

$$
\|B u\| \leqq \epsilon|u|=\epsilon\|A u\| \leqq \epsilon\|(A+B) u\|+\epsilon\|B u\|,
$$

or

$$
\|B u\| \leqq \epsilon(1-\epsilon)^{-1}\|(A+B) u\|=\epsilon(1-\epsilon)^{-1}|u|_{1} .
$$

If $\epsilon<1 / 2$ then $\epsilon(1-\epsilon)^{-1}=\delta<1$, and we can reverse the above argument to conclude that

$$
\mu_{j}(S) \leqq(1-\delta)^{-2} \mu_{j}(T) \leqq(1-2 \epsilon)^{-2} \mu_{j}(T) .
$$

Summarizing, we have

Lemma 2. If $B: K \rightarrow H$ has norm $\epsilon<1 / 2$ and $T=J\left(S_{1}^{-1}+B\right)^{-1}$ is normal, then

$$
(1-2 \epsilon)^{2} \leqq \mu_{j}(T) / \mu_{j}(S) \leqq(1-\epsilon)^{-2}
$$


3. Eigenvalue estimates for elliptic operators. Let $\Omega$ be a compact $n$-dimensional $C^{\infty}$ Riemannian manifold or manifold with boundary. Let $L^{2}(\Omega)$ be the Hilbert space with inner product $(u, v)$ corresponding to the Riemannian structure. Let $\propto$ be an elliptic operator of order $2 m$ defined on $\Omega$, having bounded measurable coefficients, and assume that the coefficients of the principal part, $Q_{0}$, are continuous on $\Omega$. Assume also that $Q$ is formally self-adjoint, $Q=Q^{\prime}$. Given a positive integer $r$, let $H^{r}(\Omega)$ be the completion of $C^{\infty}(\Omega)$ with respect to the inner product

$$
(u, v)_{r}=\sum_{|\alpha| \leqq r}\left(D^{\alpha} u, D^{\alpha} v\right) .
$$

Let $H_{0}^{r}(\Omega)$ denote the closed subspace of $H^{r}(\Omega)$ generated by smooth functions with support in the interior of $\Omega$; if $\Omega$ has no boundary, $H_{0}^{r}(\Omega)=H^{r}(\Omega)$. As is well-known, the natural injection of $H^{r}(\Omega)$ into $L^{2}(\Omega)$ is compact, for $r \geqq 1$.

Under the above assumptions on $Q$ we may define an operator $A$ in $L^{2}(\Omega)$ having domain $\mathfrak{D}(A)=H^{2 m}(\Omega) \cap H_{0}^{m}(\Omega)$, with $A u=q u$ (in the distribution sense) for $u \in D(A)$. Then $A$ is self-adjoint [cf. 3, Theorem 5], and its resolvent operator is compact. If the coefficients of $A$ were smooth, then $A$ would satisfy the hypotheses of Theorem 1 of [4]. Therefore we would have

$$
N_{A}(\lambda) \sim c(A) \lambda^{(n / 2 m)} \text { as } \lambda \rightarrow \infty,
$$

where $N_{A}(\lambda)$ denotes the number of eigenvalues of $A$ with absolute value $\leqq \lambda$. Here $c(A)$ is given by

$$
\begin{aligned}
c(A)= & (2 \pi)^{-n}(4 m s) /(n \pi) \sin ((n \pi) /(4 m s)) \\
& \cdot \int_{\Omega} \int_{R^{n}}\left(Q_{0}(x, \zeta)^{2 s}+1\right)^{-1} d \zeta d x,
\end{aligned}
$$

where $Q_{0}(x, \zeta)$ is the characteristic polynomial, and $s=[(n / 4 m)+1]$. If we let $\left\{\lambda_{j}(A)\right\}$ be the sequence of eigenvalues of $A$ repeated according to multiplicity, with $\left|\lambda_{1}(A)\right| \leqq\left|\lambda_{2}(A)\right| \leqq \cdots$, then it is easily seen that (4) is equivalent to

$$
\left|\lambda_{j}(A)\right| \sim c(A)^{-(2 m / n)} j^{(2 m / n)} \quad \text { as } j \rightarrow \infty .
$$

We want to extend this result to $A$ without smooth coefficients.

THEOREM. Let $\Omega$ be a compact $n$-dimensional $C^{\infty}$ Riemannian manifold or manifold with boundary. Let a be a formally self-adjoint elliptic operator of order $2 m$ with bounded measurable coefficients and with continuous coefficients in the principal part. Let $A$ be the realization of $a$ 
in $L^{2}(\Omega)$ having domain $H^{2 m}(\Omega) \cap H_{0}^{m}(\Omega)$. Then the eigenvalues $\left\{\lambda_{j}(A)\right\}$, with $\left|\lambda_{1}(A)\right| \leqq\left|\lambda_{2}(A)\right| \leqq \cdots$, satisfy the asymptotic formula (6), with $c(A)$ given by (5).

REMARKS. In the case of a manifold without boundary, in which case $D(A)=H^{2 m}(\Omega)$, it is not necessary to assume that $Q$ is of even order; $A$ is still self-adjoint, the formula (5) still holds for $A$ with smooth coefficients, and the following proof is unchanged.

Proof. There is a sequence $\left\{a_{\nu}\right\}$ of formally self-adjoint elliptic operators of order $2 m$, having coefficients in $C^{\infty}(\Omega)$, and such that the coefficients of the principal parts converge uniformly on $\Omega$ to the coefficients of $Q_{0}$. Let $A_{\nu}$ be the realization corresponding to $Q_{\nu}$. We can write $A_{\nu}=A+B_{\nu}$, and with respect to the norms $\|u\|_{k}$ in $H^{k}(\Omega)$ we have

$$
\left\|B_{\nu} u\right\| \leqq \delta_{\nu}\|u\|_{2 m}+C_{\nu}\|u\|_{2 m-1}, \quad u \in D(A),
$$

where the constants $\delta_{\nu} \rightarrow 0$. However there are constants $k_{\nu}$ such that

$$
C_{\nu}\|u\|_{2 m-1} \leqq \delta_{\nu}\|u\|_{2 m}+k_{\nu}\|u\|, \quad u \in H^{2 m}(\Omega) ;
$$

see the corollary of Proposition 1.1 in [2], for example. Furthermore since $A$ is closed and $D(A) \subseteq H^{2 m}(\Omega)$, there is an inequality

$$
\|u\|_{2 m} \leqq C(\|A u\|+\|u\|), \quad u \in D(A) .
$$

Combining (7), (8), and (9) we get an inequality of the form

$$
\left\|B_{\nu} u\right\|^{2} \leqq \epsilon_{\nu}^{2}\left(\|A u\|^{2}+\sigma_{\nu}\|u\|^{2}\right)=\epsilon_{\epsilon_{\nu}}^{2}\left\|\left(A+i \sigma_{\nu}\right) u\right\|^{2},
$$

where $\epsilon_{\nu} \rightarrow 0$.

Set $S=\left(A+i \sigma_{\nu}\right)^{-1}, T=\left(A+B_{\nu}+i \sigma_{\nu}\right)^{-1}=\left(A_{\nu}+i \sigma_{\nu}\right)^{-1}$. Then $R(S)$ $=\mathcal{R}(T) \subseteq H^{2 m}(\Omega)$, so $S$ and $T$ are compact. We have

$$
\mu_{j}(S)=\left|\lambda_{j}(A)+i \sigma_{\nu}\right|^{-2}
$$

and similarly for $T$. When $\epsilon_{\nu}<1 / 2$ it follows from Lemma 2 that

$$
\left(1-2 \epsilon_{\nu}\right) \leqq\left|\lambda_{j}(A)+i \sigma_{\nu}\right|\left|\lambda_{j}\left(A_{\nu}\right)+i \sigma_{\nu}\right|^{-1} \leqq\left(1-\epsilon_{\nu}\right)^{-1} .
$$

Let $c_{\nu}=c\left(A_{\nu}\right)^{-2 m / n}, c=c(A)^{-2 m / n}$. Then $\left|\lambda_{j}\left(A_{\nu}\right)\right| \sim c_{\nu} j^{2 m / n}$, so $\left|\lambda_{j}\left(A_{\nu}\right)+i \sigma_{\nu}\right| \sim c_{\nu} j^{2 m / n}$. It follows from this and (11) that

$$
\begin{gathered}
\liminf _{j \rightarrow \infty}\left|\lambda_{j}(A)\right| j^{-2 m / n} \geqq\left(1-2 \epsilon_{\nu}\right) c_{\nu}, \\
\limsup _{J \rightarrow \infty}\left|\lambda_{j}(A)\right| j^{-2 m / n} \leqq\left(1-\epsilon_{\nu}\right)^{-1} c_{\nu} .
\end{gathered}
$$

Since $c_{\nu} \rightarrow c$ and $\epsilon_{\nu} \rightarrow 0$ as $\nu \rightarrow \infty$, the desired result follows immediately from (12). 
REMARK. It is clear that, with the appropriate modification in formula (5), the theorem can be proved in the same way for elliptic systems. Furthermore it holds whenever the operator $A$ can be approximated in the above way by operators with the same domain; the boundary value problems considered here are the most obvious examples.

\section{REFERENCES}

1. S. Agmon, On kernels, eigenvalues, and eigenfunctions of operators related to elliptic problems, Comm. Pure Appl. Math. 18 (1965), 627-663.

2. R. Beals, Non-local boundary value problems for elliptic operators, Amer. J. Math. 87 (1965), 315-348.

3. F. Browder, On the spectral theory of elliptic differential operators. I, Math. Ann. 142 (1961), 22-130.

4. - Asymptotic distribution of eigenvalues and eigenfunctions for nonlocal elliptic boundary value problems. I, Amer. J. Math. 87 (1965), 175-195.

5. N. Dunford and J. T. Schwartz, Linear operators, Vol. II, Interscience, New York, 1964.

YALE UNIVERSITY AND

UNIVERSity OF CHICAgo 